\title{
Buffered electropolishing parameters on niobium sheet
}

\author{
Song Jin, ${ }^{1, *}$ Xiangyang Lu, ${ }^{1}$ Lin Lin, ${ }^{1}$ Andy T. Wu, ${ }^{2}$ and Kui Zhao ${ }^{1}$ \\ ${ }^{1}$ State Key Laboratory of Nuclear Physics and Technology, Institute of Heavy Ion Physics, School of Physics, Peking University, \\ Beijing 100871, China \\ ${ }^{2}$ Institute for SRF Science and Technology, Thomas Jefferson National Accelerator Facility, Newport News, Virginia 23606, USA
}

(Received 29 November 2009; published 7 June 2010)

\begin{abstract}
In this paper, studies on applying a new electrolyte to treat the surfaces of $\mathrm{Nb}$ sheets, which was called buffered electropolishing (BEP), were reported. Through studies of the BEP I-V characteristic and optimization of main parameters such as acid agitation, temperature, etc., much faster $\mathrm{Nb}$ polishing rate and smoother surface finish were achieved in comparison with those obtained from the conventional electropolishing (EP). The average polishing rate could reach around $2.5 \mu \mathrm{m} / \mathrm{min}$. It was over 7 times faster than that of the traditional EP. Meanwhile, the average surface mean square root roughness was around $50 \mathrm{~nm}$ over an area of $(200 \times 200) \mu \mathrm{m}^{2}$. This study shows BEP has a great potential to replace the traditional EP process and becomes a new generation of technology for treating $\mathrm{Nb}$ superconducting radio frequency cavities.
\end{abstract}

DOI: 10.1103/PhysRevSTAB.13.061001

PACS numbers: $29.20 .-\mathrm{c}$

\section{INTRODUCTION}

Surface condition plays a critical role in the performance of superconducting radio frequency (SRF) cavities [1]. Since the first SRF cavity was born, researches on niobium surface treatments have not been stopped to improve the performance. In recent years, with the buffered chemical polishing (BCP) and then electropolishing (EP) gradually becoming the chief means for SRF cavity treatments [2-4], the performance of multicell cavities has been improved greatly. However, the yield of the nine-cell cavities which can surpass the required $35 \mathrm{MV} / \mathrm{m}$ for the International Linear Collider (ILC) [5] is still not high. On the other hand, the polishing rate of the traditional EP was another problem. It was only about $0.38 \mu \mathrm{m} / \mathrm{min}$ [6]. So if a cavity is treated by the conventional EP, more than 6 hours is needed in order to remove the so-called surface damage layer of $\mathrm{Nb}$. With the long treatment period and very expensive equipment, the technology of the conventional EP made the surface treatments of SRF cavities too costly. Thus, a new method for SRF cavity treatment called buffered electropolishing (BEP) was proposed by Thomas Jefferson National Accelerator Facility (Jefferson Lab) $[7,8]$ as an alternative to try to overcome some drawbacks of the conventional EP mentioned above.

As part of the collaboration with Jefferson Lab and the study on the fabrication of multicell niobium cavity, the research on BEP was also carried out at Peking University $[9,10]$. The electrolyte of BEP consisted of $\mathrm{HF}(48 \%)$, $\mathrm{H}_{2} \mathrm{SO}_{4}(98 \%)$, and $\mathrm{C}_{3} \mathrm{H}_{6} \mathrm{O}_{3}(85 \%)$ with the volume ratio of 4:5:11. Comparing with the conventional EP, the addition of lactic acid as a buffered solution brings a lot of

\footnotetext{
*Corresponding author. jsong@pku.edu.cn
}

advantages to BEP technology [8]. However, it also made the electropolishing parameters greatly changed. In this paper, the effects of various parameters on the I-V characteristic, polishing rate, and smoothness of the BEP treated $\mathrm{Nb}$ surfaces were investigated and discussed. The goal was to evaluate the influence of various parameters to this process, as well as the technological problems involved in it. Small flat niobium samples were used in the present stage of the studies for the purpose of decreasing the cost, safety, and convenience to get isolated parameters. After the optimization of experiments, the maximum polishing rate of $4.66 \mu \mathrm{m} / \mathrm{min}$ was achieved. In the meantime, the surface rms roughness of BEP treated samples could repetitively reach around $50 \mathrm{~nm}$ over a surface area of $(200 \times 200) \mu \mathrm{m}^{2}$ as measured via a high resolution 3D profilometer by Jefferson Lab. All of the above shows that BEP had a great potential to become a new niobium surface treatment technology to replace the conventional EP. Some of the experimental results presented here have been reported in Ref. [11].

\section{EXPERIMENTAL}

\section{A. Experimental installation}

The setup of the BEP experiment is schematically shown in Fig. 1. It consisted of an electrolytic cell that had an aluminum cathode and a niobium anode (sample) immersed in a $\mathrm{HF}(48 \%), \mathrm{H}_{2} \mathrm{SO}_{4}(98 \%)$, and $\mathrm{C}_{3} \mathrm{H}_{6} \mathrm{O}_{3}(85 \%)$ mixture with the volume ratio of 4:5:11. The $\mathrm{Nb}$ anode and the pure aluminum cathode were connected to an electrical circuit through two alligator clamps. The two alligator clamps also serve as a mechanical support by fixing the electrodes to suspend on top of a polytetrafluoroethylene (PTFE) container. The circulating water or ice bath in the secondary container was used to keep the temperature of 


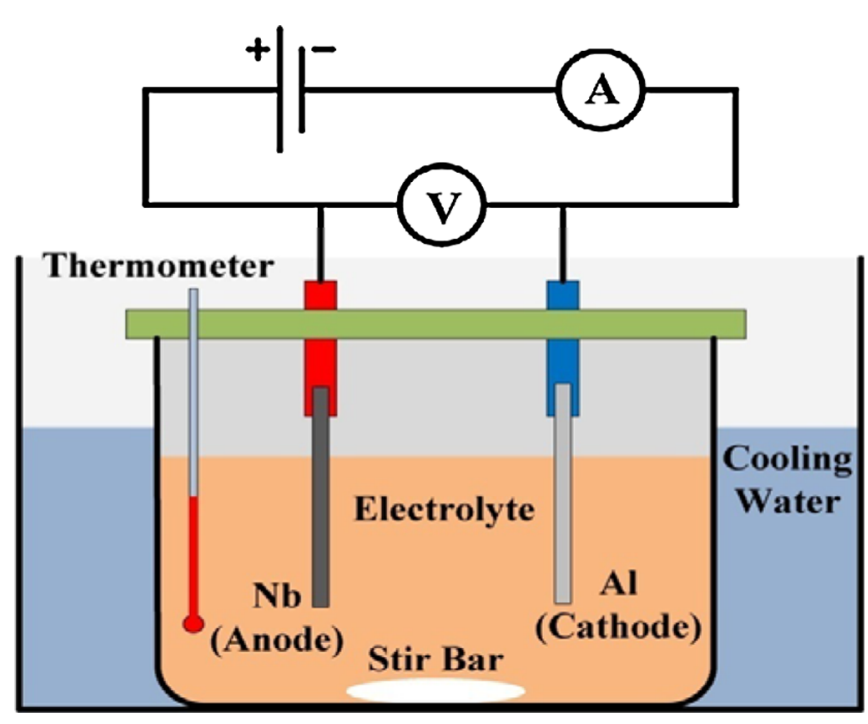

FIG. 1. (Color) Schematic installation of BEP for small samples.

the acid mixture below $35^{\circ} \mathrm{C}$ depending on the different conditions required for a particular experiment. A platinum resistor thermometer with the temperature probe protected by PTFE was immerged in the electrolyte to monitor the temperature during the BEP process. Then a magnetic stirring apparatus was used under the secondary container to control the velocity of the electrolyte flow rate. The monitor for the rotational velocity of the stir bar was a homemade apparatus according to the principle of electromagnetic induction. It mainly consisted of an induction coil and an oscilloscope. In additional, another oscilloscope was used to measure I-V characteristics employing appropriate electric circuit.

\section{B. Sample preparation}

In order to keep our small sample experiments as close as possible to the real processes used for treating $\mathrm{Nb} \mathrm{SRF}$ cavities, high-purity niobium samples were used in the BEP experiments. Their residual resistance ratio was above 300 . With the dimension of $10 \mathrm{~mm} \times 70 \mathrm{~mm}$, the samples were cut out from a $3 \mathrm{~mm}$ thick niobium sheet used for fabricating SRF cavity, so the physical properties of the samples were the same as those used for fabricating $\mathrm{Nb}$ SRF cavities. Then referring to the pretreatment process of EP [12], the niobium samples were first cleaned by acetone and isopropyl alcohol; subsequently, it was ultrasonically cleaned in soapy water; after that it was rinsed by ultrasonic cleaning with deionized water; and finally, after blowing away the remaining water by dry nitrogen gas and weighing, it could be used for the BEP experiment.

\section{RESULTS AND DISCUSSION}

\section{A. I-V characteristic of BEP for niobium samples}

The electrochemical process must be tweaked to the optimal I-V combination in order to obtain the best $\mathrm{Nb}$

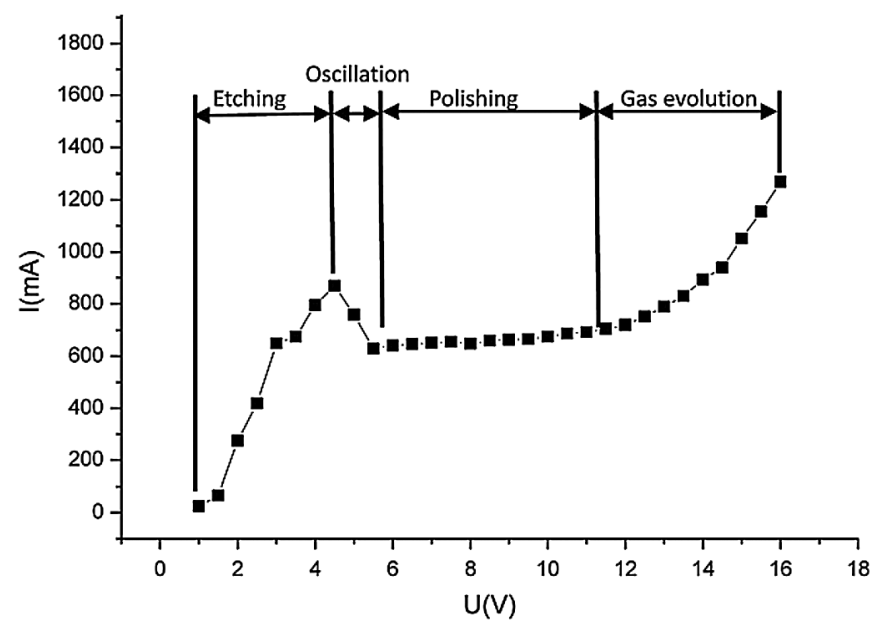

FIG. 2. The typical I-V curve for buffered electropolishing.

surface. So, studies on the I-V characteristic of BEP also became necessary. Figure 2 shows an I-V curve for BEP of niobium sample. Like the typical electropolishing I-V characteristic, four regions corresponding to etching, periodic oscillation of anode current density, polishing, and gas evolution on the anode surface were identified depending on the potential applied. Initially, the current density increased linearly with the voltage until reaching the maximum value. At this region, the behavior was dominated by ohm resistance in the electrolyte. Then, current began to decrease with the oscillation until reaching a steady plateau. In this region, a relatively strong oxide layer was built up on the surface, which could be considered as a capacitor. Thus the current began to oscillate. Over a horizontal range, the current density was nearly independent of the applied voltage and the surface was polished. For even higher voltages, gaseous oxygen is set free at the anode and the oxygen bubbles result in discontinuities on the surface.

However, as to the real polishing process, the conditions are more complicated, and not all the measured I-V curves have the same shape. The detailed shape of the curve varies with the different conditions under study. It depends not only on the electrolyte flow rate, but also on temperature and other conditions.

Figure 3 is the development of the I-V curve with a different flowing speed of electrolyte at the temperature of $25^{\circ} \mathrm{C}$. This investigation was necessary because acid agitation was an indispensable means in electrochemical polishing in order to keep a stable material removal in time, especially in the case of high viscosity of the acid mixtures in BEP and for the irregular cavity geometry. It would become an important parameter in order to get an optimal polishing rate and smooth $\mathrm{Nb}$ surface. Here, the flowing speeds of electrolyte were indicated by the rotation rates of the magnetic stirring bar. With the static electrolyte, the I-V curve had a long polishing plateau. But the limiting current density was the smallest of all. When the flow rate 


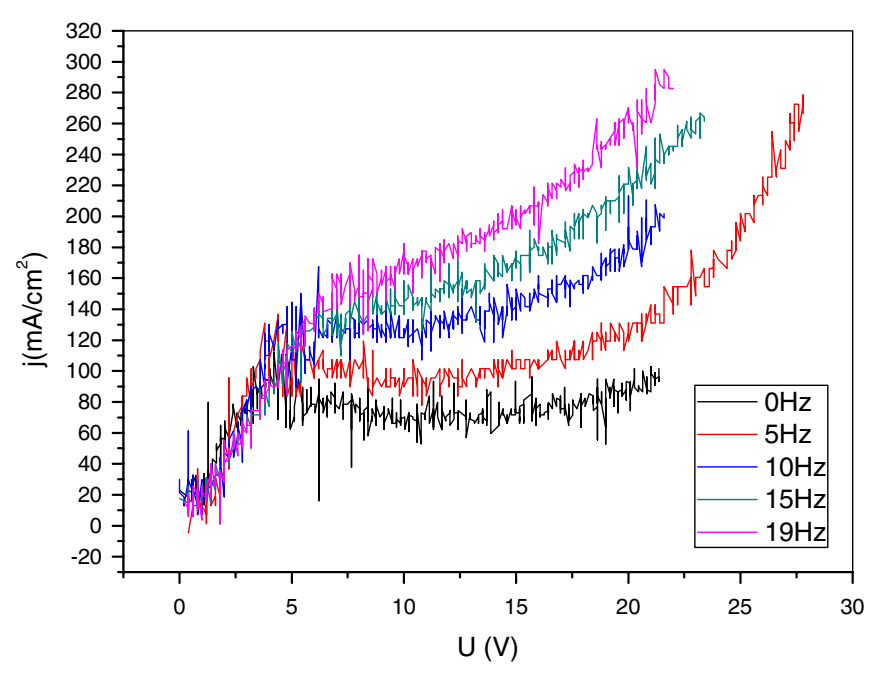

FIG. 3. (Color) Development of the I-V curve with different rotational speed of the stirring bar at $25^{\circ} \mathrm{C}$.

of the electrolyte became faster, the width of the polishing plateau changed very little. However, the limiting current density continued to increase. Then, when the rotational speed of the magnetic bar was above a special value, about $15 \mathrm{~Hz}$, the horizontal parts of curves began to become a slanted upward curve. As to this phenomenon, we thought it was not a symbol of the maximum electrolyte flow rate we could use, although the polishing plateau was not as clear as those at the lower flowing speed. Oppositely, we thought it represented that the flow rate of electrolyte was just fast enough to carry the complex compound of the niobium oxide layer around the niobium sample surface away. That fast flow rate was necessary especially to the high viscosity BEP acid solution because the high viscosity would make the anodic compound oxide layer [13-15] have a larger voltage drop as compared with that of a common acid solution. So, the faster electrolyte flowed, the more external complex composition of the compound oxide layer could be carried away, leading to an enhanced electric field strength in the compact layer near the anode surface. We believe that this stronger electric field is responsible for the higher polishing rate during BEP. In the mean time, the electric field difference between protrusions and well is also enlarged which would make the surface much smoother. The following experiment result will confirm it. As to the trend of limiting current density increasing with the faster electrolyte flowing speed, it was also consistent with our judgment at the beginning. Because the compound oxide layer on the surface of the sample would take a lot of voltage, the faster the electrolyte flowed, the less sticky layer would be left and the smaller voltage it would be take. And the curve would move up.

Apart from acid agitation, we found the I-V curve was also sensitive to the temperature in the BEP process. Figure 4 shows the I-V curves with different temperatures. They were measured at the temperatures of $12.5,16.5$, and

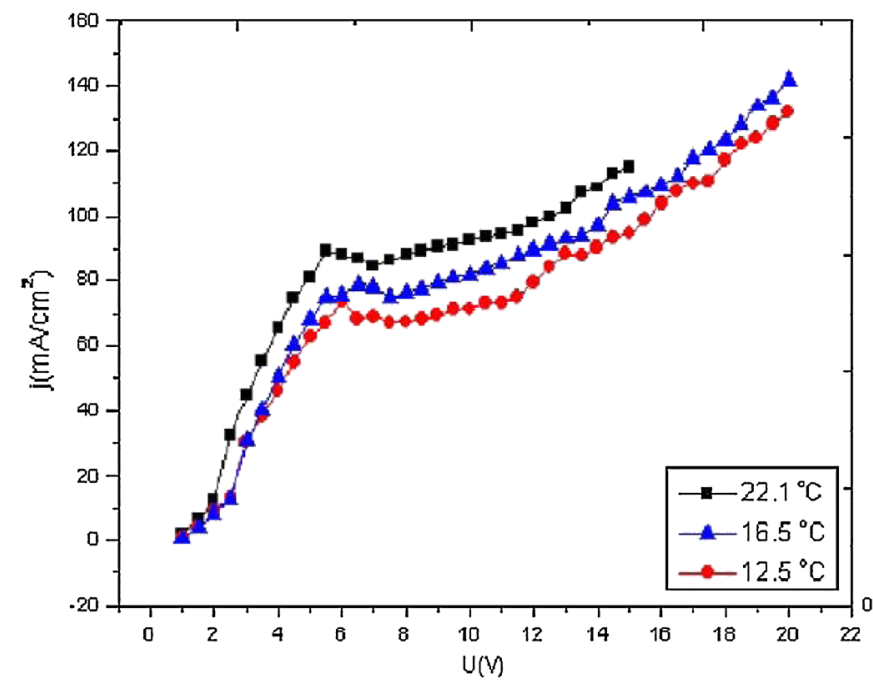

FIG. 4. (Color) Development of the I-V curve with different temperature with the stirring frequency of $11 \mathrm{~Hz}$.

$22^{\circ} \mathrm{C}$ with the same stirring frequency of $11 \mathrm{~Hz}$. In the part of the polishing plateau regions, it was similar to those with different flowing speeds of electrolyte above. With an increasing temperature, the limiting current density also increased. This could be understood by the theory that the higher temperature could increase the mobility of the ions in the electrolyte, which made the chemical reaction near anode surface faster. However, as to the part of etching region, we could see the different effects of temperature and electrolyte flow rate on the I-V characteristic. With different electrolyte flow rate, the slopes of the I-V curve of this part were nearly the same. But when the temperature is varied, the slopes were various. With higher temperature, the slope of the I-V curve of this region became much sharper. This experimental result could be understood in the following way: Agitation could not change the physical or chemical property of the electrolyte, however temperature could. The simplest explanation for this phenomenon was that, within certain range, the higher temperature could lower the viscosity of BEP electrolyte. So the resistance of the electrolyte decreased, and the curve in the etching region would be sharper. However, a detailed mechanism might not be so simple, and further study is needed. But here the result of the trend of limiting current density development with temperature in BEP was our main concern since the limiting current density represented the polishing rate of this technology, which will be studied in the following part of this paper.

\section{B. Effect of parameters on polishing rate}

In the process of niobium cavity treatment, the polishing rate is one of the most important parameters. A high polishing rate can greatly reduce the time of cavity fabrication and further decrease the cost of the SRF cavity. In addition, to shorten the process time of the niobium cavity 


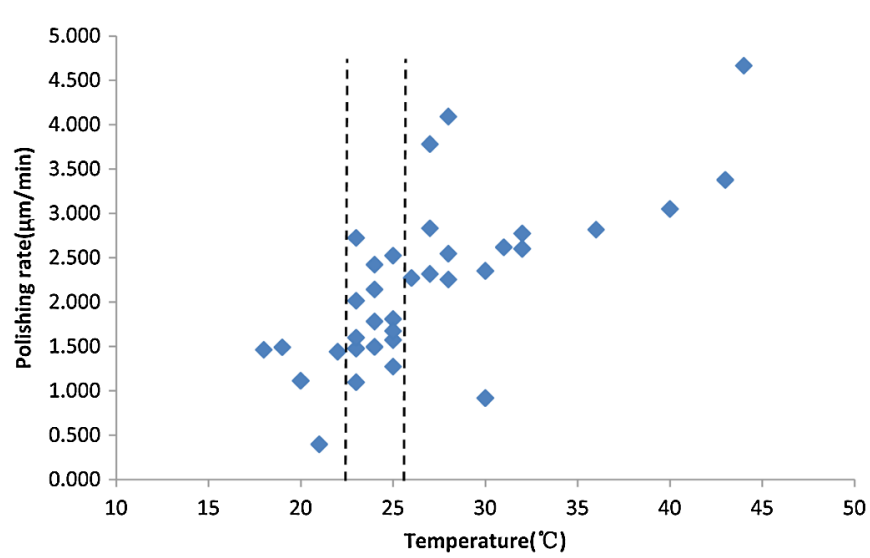

FIG. 5. (Color) The effect of temperature on polishing rate from all the experiments including those with different acid flow rates.

was also the demand of the ILC [5] which will need nearly twenty thousand nine-cell cavities for this great project.

Figure 5 is a statistical result about the effect of temperature on polishing rate. This figure is a collection of all the experiments done at PKU, including those obtained at different acid flow rates. The whole figure can be roughly divided into three regions by temperature according to the polishing rate. In the first region, the temperature is below $23^{\circ} \mathrm{C}$. In this part, the maximum polishing rate is only about $1.5 \mu \mathrm{m} / \mathrm{min}$. The second region is between $23^{\circ} \mathrm{C}$ and $26^{\circ} \mathrm{C}$. In this region, the polishing rates are distributed from about 1.0 to $2.7 \mu \mathrm{m} / \mathrm{min}$. In the last region, the temperature is above $26^{\circ} \mathrm{C}$. As shown in the figure, except one point, all the other polishing rates are more than $2.3 \mu \mathrm{m} / \mathrm{min}$, and the polishing rate does not increase obviously with increasing temperature. We can conclude that, if we want to get a high polishing rate, a temperature higher than $23^{\circ} \mathrm{C}$ is needed. If the temperature we choose is in the second region, other parameters such as agitation, voltage, and so on should be taken into consideration to improve the polishing rate. If the temperature we choose for polishing is in the third region, the polishing rate is high enough and therefore should not be a problem. However, in this case, especially above $35^{\circ} \mathrm{C}$, the reaction is hard to control [12], and more impurities including oxides may be brought into the anode surface.

Apart from temperature, electrolyte flow rate can also affect the polishing rate. Although it is not as obvious as the effect of temperature, it still can be divided into two regions by the flow rate of electrolyte as shown in Fig. 6 . Here, the data were also from all the experiments including different temperatures. The flow rate of electrolyte is represented by rotational frequency of the magnet stirring bar. We can see, in the left region of the black line, the rotational frequency of the stirring bar is below $15 \mathrm{~Hz}$, and most of the polishing rates are concentrated from about 0.5 to $2.2 \mu \mathrm{m} / \mathrm{min}$. In the right region, where the rotational frequency is above $15 \mathrm{~Hz}$ (include $15 \mathrm{~Hz}$ ), the minimum polishing rate is about $1.5 \mu \mathrm{m} / \mathrm{min}$ and the maximum

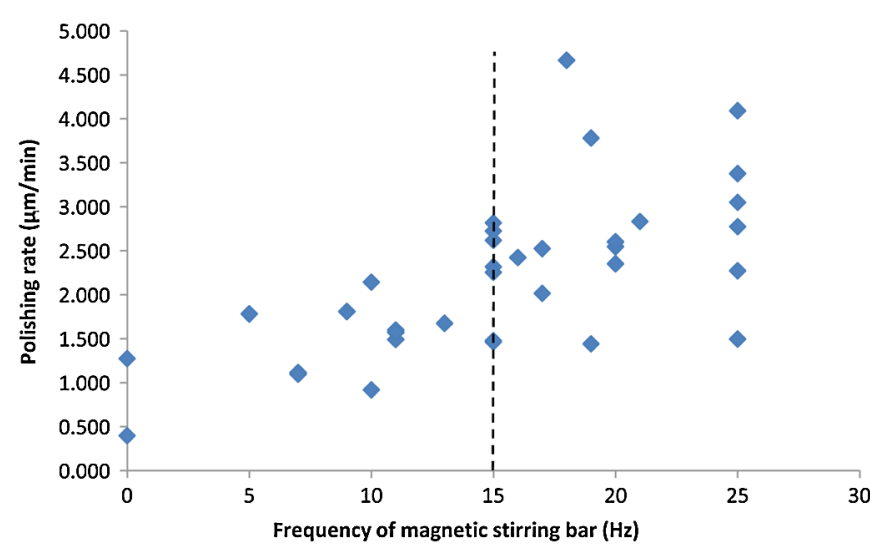

FIG. 6. (Color) The effect of flow rate of electrolyte on polishing rate from all the experiments including those with different temperatures.

polishing rate can reach about $4.7 \mu \mathrm{m} / \mathrm{min}$. Although the data points are a little scattered, we concluded that if a high polishing rate is needed, the rotational frequency of magnetic stirring bar should be above $15 \mathrm{~Hz}$. This conclusion is also identical with the analysis in the part of the I-V characteristic. Another explanation for this conclusion is that a faster electrolyte flow rate is caused by making the compact oxide film on the anode surface thinner [16,17]. Since the polishing process is governed by a diffusion process through the compact oxide film, a thinner film allows the ions to get through it in a much easier way. Therefore it speeds up the polishing rate.

Another part of content about the polishing rate of BEP was the relationship between polishing rate and current density. Figure 7 shows this relationship and the data were still from all the experiments with different temperatures and flow rate rates. As we see, the polishing rates increased linearly with the current density while the slope coefficient is around 0.0143 . According to Faraday's law of electroly-

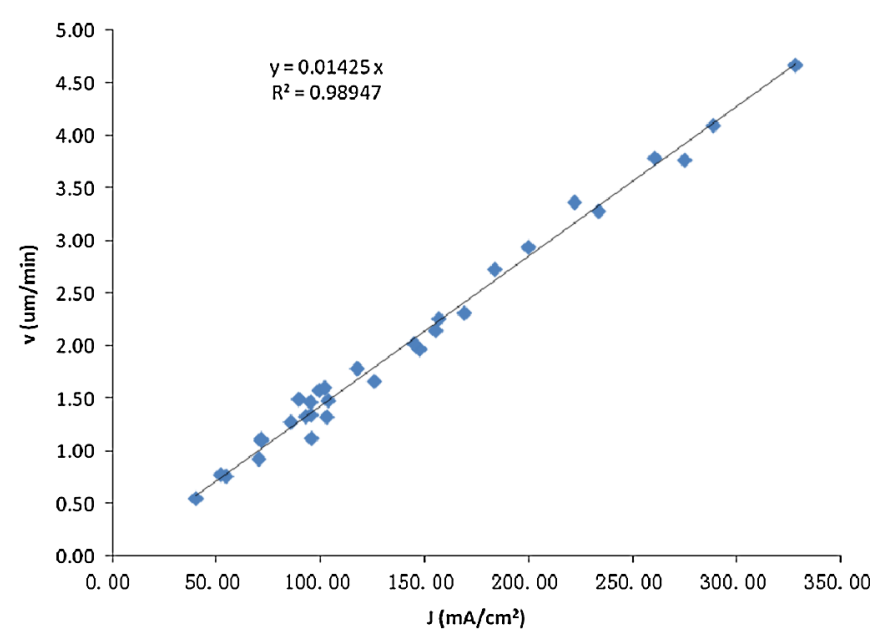

FIG. 7. (Color) The validation of Faraday's law of electrolysis in BEP. 
sis, it shows that the main electrochemical reaction in BEP should belong to typical Faraday reaction, and the valence of niobium after the reaction should be the same because of the observed linear relationship between these two parameters. From Fig. 7, we could speculate the valence of the niobium ion participating in the reaction in BEP according to the value of the slope in the figure in the following way: First, we assumed the valence of niobium in the reaction was $5+$. If the polishing current density is $1 \mathrm{~A} / \mathrm{cm}^{2}$, there will be $1 \mathrm{C}$ charge in the reaction, and the mass of niobium in reaction will be

$$
\begin{aligned}
m & =\frac{\left(6.28 \times 10^{18}\right)}{5} \times \frac{1}{6.02 \times 10^{23} / \mathrm{mol}} \times 93 \mathrm{~g} / \mathrm{mol} \\
& =19.4 \times 10^{-5} \mathrm{~g} .
\end{aligned}
$$

This means for the current density $1 \mathrm{~A} / \mathrm{cm}^{2}$, the thickness polished in one second will be

$$
d=\frac{19.4 \times 10^{-5} \mathrm{~g}}{8.66 \mathrm{~g} / \mathrm{cm}^{3} \times 1 \mathrm{~cm}^{2}}=2.24 \times 10^{-5} \mathrm{~cm} .
$$

Usually, we use micrometer per minute to describe polishing rate and milliampere per square centimeter to describe current density in electropolishing. So, for the current density of $1 \mathrm{~mA} / \mathrm{cm}^{2}$, the polishing rate is

$$
\begin{aligned}
v= & 2.24 \times 10^{-5} \mathrm{~cm} / \mathrm{s} \times \frac{1}{1000} \times 10^{4} \mu \mathrm{m} / \mathrm{cm} \\
& \times 60 \mathrm{~s} / \mathrm{min} \\
= & 0.0134 \mathrm{~m} / \mathrm{min} .
\end{aligned}
$$

Then we can get the slope coefficient of the polishing rate vs current density curve, which was 0.0134 . This is only about $5.6 \%$ less than the observed value which is shown in Fig. 7. That is to say, this theoretical value is quite close to the real situation of reaction, and most niobium ions indeed took part in the reaction with the valence of $5+$. As to the reason why the measured slope value in experiment was a little higher than our calculated value, we thought it could be that few lower valence niobium ions or other oxidizer took part in the reaction.

\section{Effect of removed thickness on surface roughness in BEP}

Because of the large number of variables involved in the fabrication and conditioning of the cavities, it is not very obvious how to establish a correlation between surface roughness and cavity performance. However, in general, the smoother the surface is, the higher the probability will be to obtain a better performing cavity. To achieve the optimal rf performance, the surface of the cavity must be as close as possible to the ideal one [1]. In our BEP experiment, the best surface rms roughness can be close

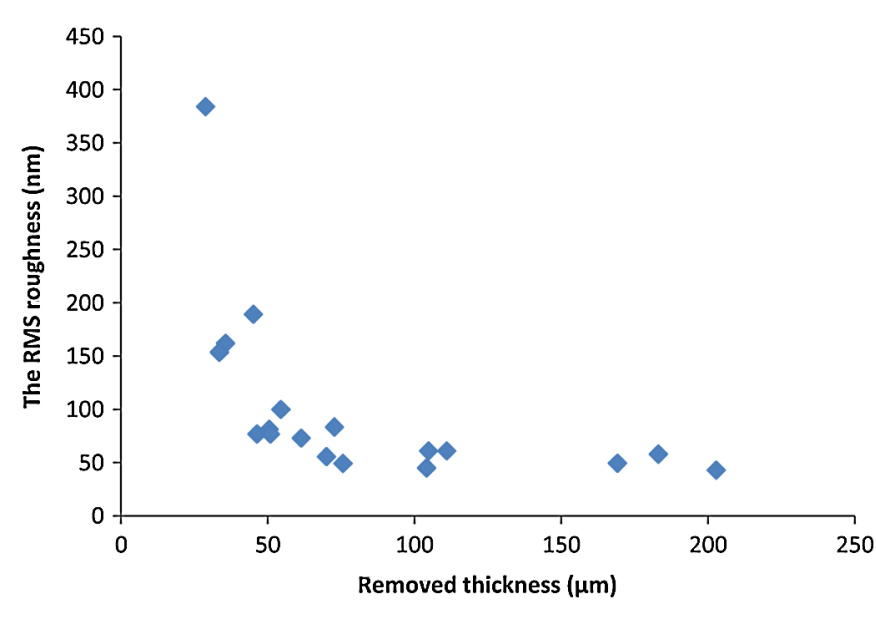

FIG. 8. (Color) Relationship between the roughness and removed thickness.

to $21 \mathrm{~nm}$ which was measured in an area of $(200 \times$ 200) $\mu \mathrm{m}^{2}$ at Jefferson Lab.

Figure 8 shows the relationship between roughness and removed thickness in the BEP process. The samples were random selected with different polishing temperatures and electrolyte flowing speeds. However, we can find that the rms roughness still has a strong dependence mainly on the removed thickness in BEP. As shown in Fig. 8, when the removed thickness is less than $50 \mu \mathrm{m}$ the rms roughness decreases rapidly with the increase of removed thickness from nearly 400 to $100 \mathrm{~nm}$. Then, from the removed thickness 50 to $75 \mu \mathrm{m}$ the change of rms roughness becomes slow. After that, when the removed thickness is above $75 \mu \mathrm{m}$, a long nearly horizontal region with the rms roughness around $50 \mathrm{~nm}$ appears. This provides very useful information for us to further study the cavity BEP processing. If we want to get a smooth surface repetitively, the minimum removed thickness about $75 \mu \mathrm{m}$ is needed. It can be explained by the theory of electropolishing proposed by Jacquet theory [12]. In the polishing area, a viscous layer of anodic dissolution products forms during the BEP process. With respect to the bulk of the electrolyte, this layer has higher viscosity and greater electrical resistivity. The thickness of the liquid insulating layer is greater in crevices than on projections. Thus, the current density on projections will be much higher than in crevices, and projections dissolve more rapidly than crevices. So, it produces the altitude difference between projects and crevices less and less with the increase of average removed thickness.

\section{Surface topography and chemical composition after BEP}

Figure 9 shows the surface topography of niobium samples with different instruments. Figure 9(a) was the optical picture of BEP treated samples. Obviously, each sample can be divided into two parts. The shining part is 

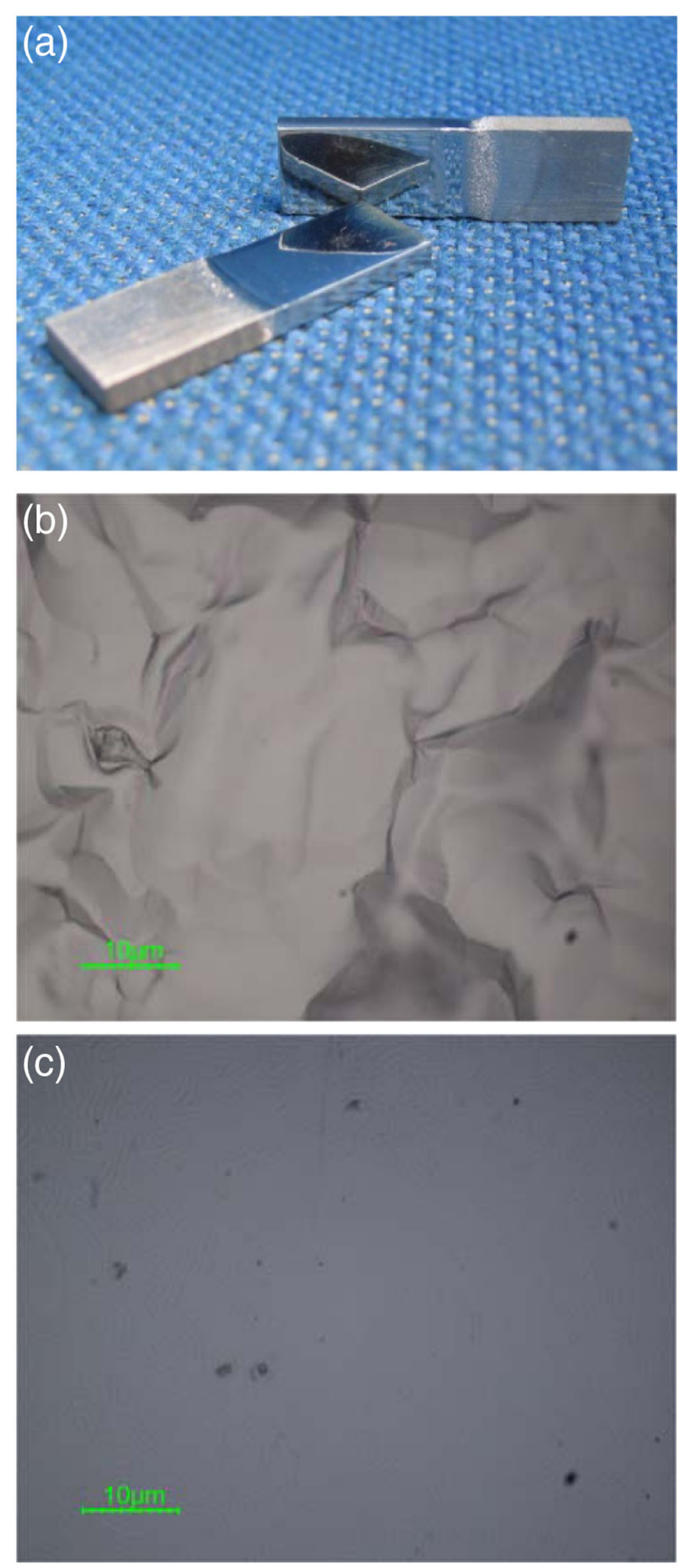

FIG. 9. (Color) CCD images of niobium sheet surface. (a) Photo of the $\mathrm{Nb}$ samples treated by BEP. (b) Typical fine grain $\mathrm{Nb}$ surface after BCP treated as viewed by metallographic optical microscope (MOM). (c) Typical MOM image of BEP treated $\mathrm{Nb}$ surface.

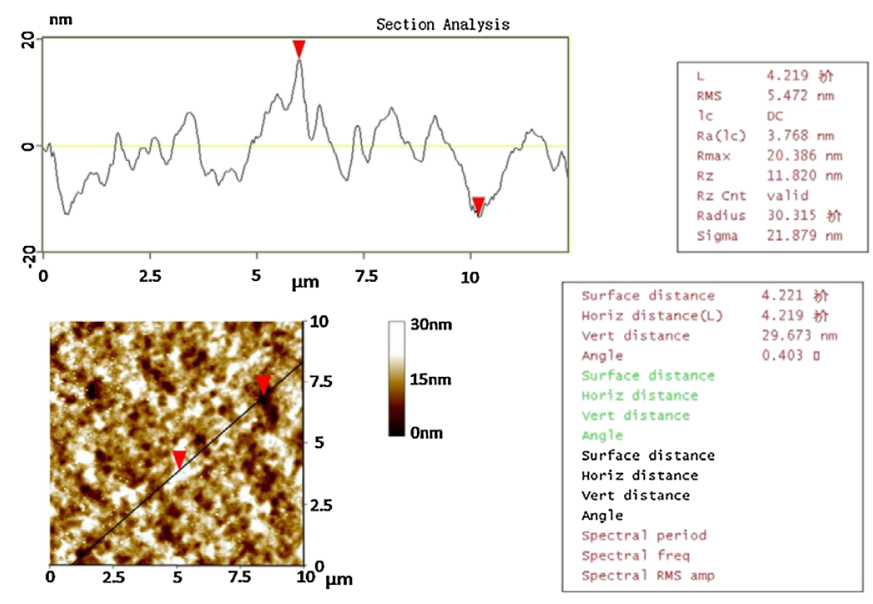

FIG. 10. (Color) The AFM images of the niobium sample surface treated by BEP.

treated by BEP, while the other part of the sample is the original surface. We can see the reflection of the other sample clearly in the shining part like the case in a mirror. Figures 9(b) and 9(c) were the surface topographies observed by metallographic optical microscope (MOM), respectively, corresponding to the BCP treated surface and the BEP treated surface. They are the images magnified to 1000 times. The difference between the two pictures could be visualized easily. Actually, the rms roughness of the niobium surface treated by BEP will be about 20 times better than those treated by BCP [6]. In addition, in the process of taking pictures of them with MOM, sometimes it is much harder to focus on the BEP treated surface since it is so smooth as compared with that on BCP treated one. Figure 10 was the image of the BEP treated niobium sample surface measured by atomic force microscope (AFM). It shows the rms surface roughness was less than $10 \mathrm{~nm}$ with the area of $(10 \times 10) \mu \mathrm{m}^{2}$. However, it could not represent the real surface smoothness because of the small scanned area. Figure 11 is a picture scanned by a high resolution 3D profilometer on the best BEP treated sample over a $(200 \times 200) \mu \mathrm{m}^{2}$ area at Jefferson Lab. With the rms roughness of $21 \mathrm{~nm}$, it is hard to see any surface variation from it. As to the average rms roughness

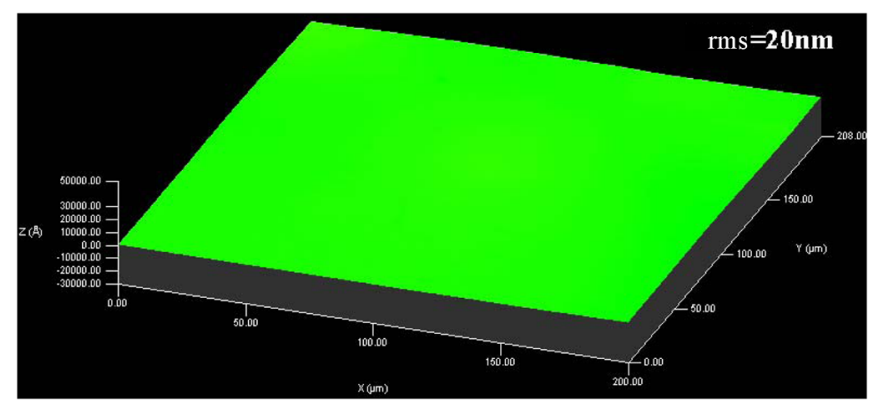

FIG. 11. (Color) The picture of the best BEP treated niobium surface scanned by high resolution $3 \mathrm{D}$ profilometer with the area of more than over $(200 \times 200) \mu \mathrm{m}^{2}$. 


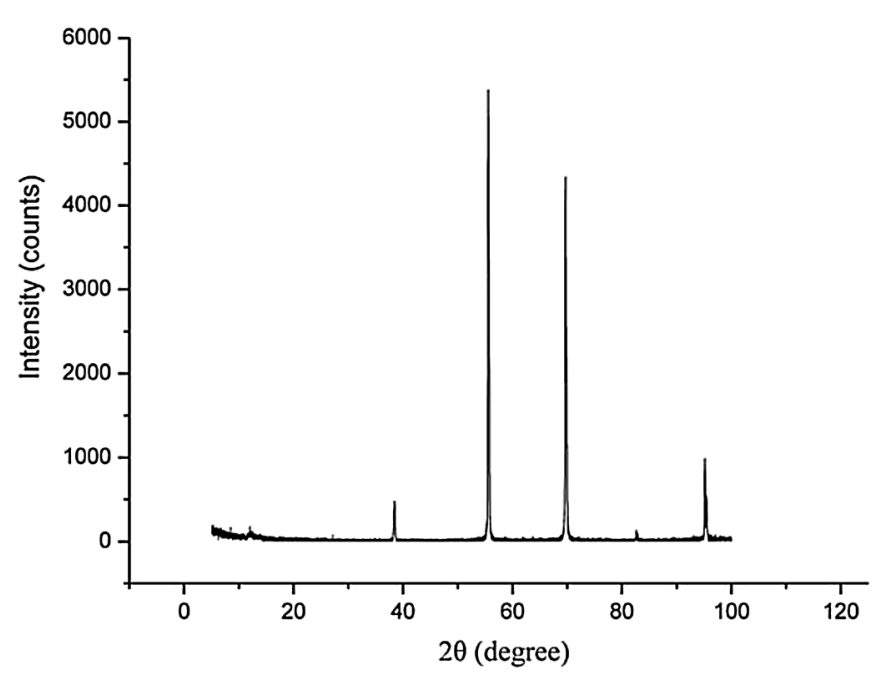

FIG. 12. Typical XRD diffraction pattern of BEP treated $\mathrm{Nb}$ samples.

of $50 \mathrm{~nm}$, it is still improved by 5 times in comparison with the rms roughness of $250 \mathrm{~nm}$ of the traditional EP.

Furthermore, a preliminary result about the chemical composition of BEP treated $\mathrm{Nb}$ samples was reported here. Although the real causes which prevent the Nb SRF cavities from reaching the niobium intrinsic limit are still mysterious and unresolved until today, generally speaking the possibility for getting a good performance SRF $\mathrm{Nb}$ cavity will be higher if the surface impurity contained is less. Thus, the knowledge about the chemical composition of BEP treated $\mathrm{Nb}$ surface is also attractive. Figure 12 is the typical $\mathrm{x}$-ray diffraction (XRD) diffraction pattern of the BEP treated samples. As we see, there are five peaks in this pattern. Except the peak at about 82 degrees and some noise from 5 to 12 degrees which are caused by the XRD facility, the whole pattern including the other four peaks is very clear. Comparing with the standard spectrum, the observed five peaks correspond to the niobium peaks very well. In other words, we cannot find any impurities brought to the niobium surface through BEP treatment via $\mathrm{XRD}$ measurement. It only represented that there were no noticeable impurities brought in, and the overwhelming majority composition in the surface was niobium within the measurement ability of XRD. More precise and quantitative analysis about composition and performance for the BEP treated samples employing other measurement techniques and the comparison with the results obtained from $\mathrm{Nb}$ samples treated by $\mathrm{BCP}$ and $\mathrm{EP}$ will be carried out in the near future.

\section{CONCLUSION}

In this paper, $\mathrm{BEP}$ technology for treating small $\mathrm{Nb}$ flat samples was systematically studied. Through investigation and discussion of effects of parameters on the I-V characteristic, polishing rate, and surface roughness, the optimum results were obtained. The polishing rate could repetitively reach around $2.5 \mu \mathrm{m} / \mathrm{min}$, while the surface rms roughness could repetitively reach around $50 \mathrm{~nm}$ which was measured in an area over $(200 \times 200) \mu \mathrm{m}^{2}$ using a precision 3D profilometer at Jefferson Lab. Comparing with traditional EP, the average BEP polishing rate is more than 7 times faster, and the surface rms roughness is decreased by more than 5 times. The result of the maximum polishing rate of $4.7 \mu \mathrm{m} / \mathrm{min}$ and the best roughness of $21 \mathrm{~nm}$ also indicate that the BEP technology is improvable. All the above show that the BEP technology has a great potential to replace the traditional EP process and becomes a new generation of technology for treating niobium superconducting rf cavities. Besides, the surface chemical compositions of BEP samples were studied by $\mathrm{XRD}$. It is shown that BEP did not induce some bad compounds to the treated surfaces within the measurement limit of XRD.

\section{ACKNOWLEDGMENTS}

We would like to acknowledge Dr. Larry Philips from Jefferson Lab for providing some niobium sheets for our experiment. This work was supported by The National Basic Research Program of China (2002CB713600).

[1] H. Padamsee, J. Knobloch, and T. Hays, $R F$ Superconductivity for Accelerators (Wiley, New York, 1998).

[2] K. Saito et al., in Proceedings of the 8th Workshop on RF Superconductivity, Abano Terme, Italy, 1997, Vol. IV, p. 795, SRF97D02.

[3] E. Kako et al., in Proceedings of the 9th Workshop on RF Superconductivity, Santa Fe, NM, 1999, Vol. I, p. 179, TUP011.

[4] B. Visentin, in Proceedings of the 11th Workshop on RF Superconductivity, Lübeck/Travemünde, Germany, 2003, TuO01.

[5] ILC Global Design Effort and World Wide Study, International Linear Collider Reference Design Report, http://ilcdoc.linearcollider.org/record/6321/files/ ILC_RDR_Volume_3-Accelerator.pd

[6] A. T. Wu et al., Appl. Surf. Sci. 253, 3041 (2007).

[7] J. R. Delayen et al., in Proceedings of the 10th Workshop on RF Superconductivity, Tsukuba, Japan, 2001, p. 499, PT014.

[8] A. T. Wu, in Proceedings of the 11th Workshop on RF Superconductivity, Lübeck/Travemünde, Germany, 2003 (Ref. [4]), ThP13.

[9] Song Jin et al., in Proceedings of the 13th Workshop on SRF, Beijing, China, 2007, WEP86.

[10] Erdong Wang et al., in Proceedings of the 13th Workshop on SRF, Beijing, China, 2007 (Ref. [10]), WEP65.

[11] Song Jin et al., in Proceedings of the 14th Workshop on SRF, Berlin, Germany, 2009, THPPO097.

[12] Final Surface Preparation for Superconducting Cavities, TESLA Technology Collaboration TTC-Report No. 200805, http://flash.desy.de/sites/site_vuvfel/content/e403/ 
e1644/e2271/e2272/infoboxContent2354/TTCReport2008-05.pdf.

[13] P. A. Jacquet, Trans. Electrochem. Soc. 69, 629 (1936).

[14] A. Hickling and J. K. Higgins, Trans. Inst. Met. Fin. 29, 274 (1953).
[15] H. F. Walton, J. Electrochem. Soc. 97, 219 (1950).

[16] W. C. Elmore, J. Appl. Phys. 10, 724 (1939).

[17] T. P. Hoar and J. A. S. Mowat, Nature (London) 165, 64 (1950). 